\title{
Hepar Lobatum Carcinomatosum Associated with Metastatic Rectal Carcinoma: An Unusual Cause of Liver Dysmorphy
}

\author{
Zafer Teke $^{\mathrm{a}}$ Gurel Nessar $^{\mathrm{a}}$ Saba Kiremitci ${ }^{\mathrm{b}}$ Erol Aksoy ${ }^{\mathrm{a}}$ Orhan Hayri Elbir ${ }^{\mathrm{a}}$ \\ Departments of a Gastroenterological Surgery and ${ }^{b}$ Pathology, Turkey Yuksek Ihtisas Teaching and Research Hospital, \\ Ankara, Turkey
}

\section{Key Words}

Liver $\cdot$ Hepar lobatum $\cdot$ Hepar lobatum carcinomatosum •

Liver metastasis • Metastatic carcinoma $\cdot$ Rectal carcinoma Chemotherapy

\begin{abstract}
Objective: The aim of this study was to present a case of hepar lobatum resulting from metastatic rectal carcinoma. Clinical Presentation and Intervention: A 50-year-old man presented with a 2-year history of bleeding per anum, tenesmus, malaise and weakness. Initially, the patient received neoadjuvant chemoradiotherapy followed by abdominoperineal resection of the rectum. Abdominal computed tomography showed lobar enlargement and lobulated contour, mainly in the left lobe of the liver, but no primary or metastatic lesions were detected. Laparotomy revealed an irregularly lobulated hepatic deformity. Liver biopsy showed a necrotic tumor growth from adenocarcinoma of the rectum in subcapsular localization of the liver. Conclusion: This case showed a patient with hepar lobatum carcinomatosum caused by metastatic rectal carcinoma. The report further highlights the need for clinicians and surgeons to keep in mind the possibility of hepar lobatum carcinomatosum while caring for rectal carcinoma patients, especially when
\end{abstract}

the lobulated contour of the liver is detected at preoperative imaging studies or when the coarsely lobated liver is encountered during surgery for carcinoma of the rectum.

Copyright $\odot 2010$ S. Karger AG, Basel

\section{Introduction}

Hepar lobatum refers to liver deformity, including an irregular, lobulated contour with crevices and linear depressions, seen in patients with tertiary syphilis, resulting from healing gummas and scar contraction [1, 2]. Many cases with the classic appearance of hepar lobatum have been described [3-7], especially in association with metastatic breast carcinoma. Recently, we encountered a liver dysmorphy not related to syphilis in a patient with metastatic rectal carcinoma.

\section{Clinical Presentation and Intervention}

A 50-year-old man presented with a 2-year history of bleeding per anum, tenesmus, malaise and weakness. The patient had experienced increasing constipation and a sense of obstruction in the rectum for 3 months. His medical history showed that the patient had undergone an open hemorrhoidectomy and a hydroce-

\section{KARGER}

Fax +41613061234 E-Mail karger@karger.ch www.karger.com
(C) 2010 S. Karger AG, Basel

1011-7571/11/0201-0093\$38.00/0

Accessible online at:

www.karger.com/mpp
Zafer Teke

Yesilkoy Mah. Yesilada Sitesi, 544 Sok. No. 3

A-Blok, Kat 4, Daire 9

TR-20055 Servergazi, Denizli (Turkey)

Tel. +90 542264 4046, Fax +90 312312 4120, E-Mail zteke_md@yahoo.com 
Fig. 1. Axial CT of the liver showing a homogeneous liver with a nodular contour in the left lobe.
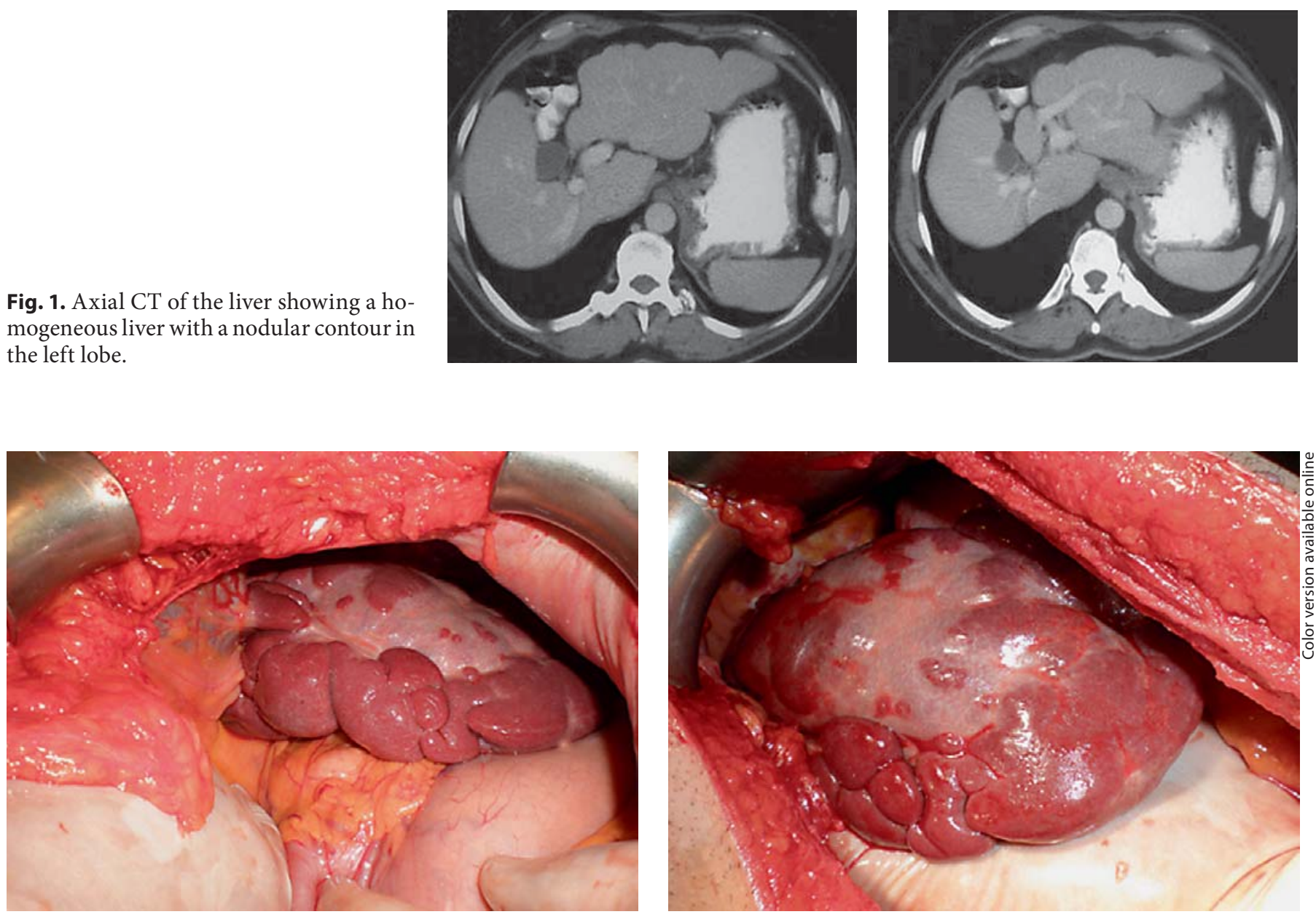

Fig. 2. Gross appearance of the liver at operation. Note the coarsely lobulated surface.

lectomy 10 and 13 years previously, respectively. The family and social histories were noncontributory.

On admission, physical examination was normal, but hematological examination showed a hemoglobin count of $11.0 \mathrm{~g} / \mathrm{dl}$ (normal: $13.6-17.2 \mathrm{~g} / \mathrm{dl}$ ) and a hematocrit of $33.4 \%$ (normal: $39.5-$ $50.3 \%$ ). The level of carcinoembryonic antigen was $48.9 \mathrm{ng} / \mathrm{ml}$ (normal range: $0-4.3 \mathrm{ng} / \mathrm{ml}$ ). Preoperative syphilis serum tests, including venereal disease research laboratory test, treponema pallidum hemagglutination assay and fluorescent treponemal antibody absorption test were negative. Colonoscopy revealed a large, fragile polypoid lesion extending from the anal canal to the rectum. Biopsy results showed rectal adenocarcinoma. Abdominal computed tomography (CT) showed lobar enlargement and a lobulated contour, mainly in the left lobe of the liver (fig. 1). The portal and hepatic veins were patent. No primary or metastatic lesions were detected in the liver. The patient received preoperative chemoradiotherapy. Radiotherapy consisted of a total of 4,500 cGy delivered in 28 fractions of 180 cGy 5 times weekly to the pelvis. During the first and fifth weeks of radiotherapy, 5fluorouracil (5-FU) was given as a 120-hour continuous infusion at a dose of $225 \mathrm{mg} / \mathrm{m}^{2}$ per day. A formal abdominoperineal resec- tion of the rectum was performed 6 weeks after the completion of neoadjuvant chemoradiotherapy. On exploration of the abdomen, the liver manifested the gross appearance of hepar lobatum. The surface was coarsely lobated (the so-called 'potato liver') (fig. 2), and the capsule was slightly thickened. The surface of the lobulated areas was smooth. More nodular masses were concentrated on the anterior margins of segments III and IV A of the liver. A lesion measuring $1 \times 1 \mathrm{~cm}$, on the surface of segment $\mathrm{V}$ of the liver, was excised locally, and the specimen was sent for frozen section examination. The result was reported to be liver metastasis from adenocarcinoma.

Histological examination of the liver biopsy showed a necrotic tumor growth in subcapsular localization of the liver (fig. 3a). There were scattered foci of dilatation of portal veins and sinusoids (fig. 3b). There were no granulomas, giant cells or features indicating coexisting chronic hepatitis. The Ziehl-Neelsen stain for acid-fast organisms, the periodic acid-Schiff and Grocott methenamine silver stains for fungal and protozoal infections were negative. Schistosome ova were not present in noncancerous areas of the liver tissue. Histopathological examination of the APR specimen revealed moderately differentiated rectal adenocarci- 

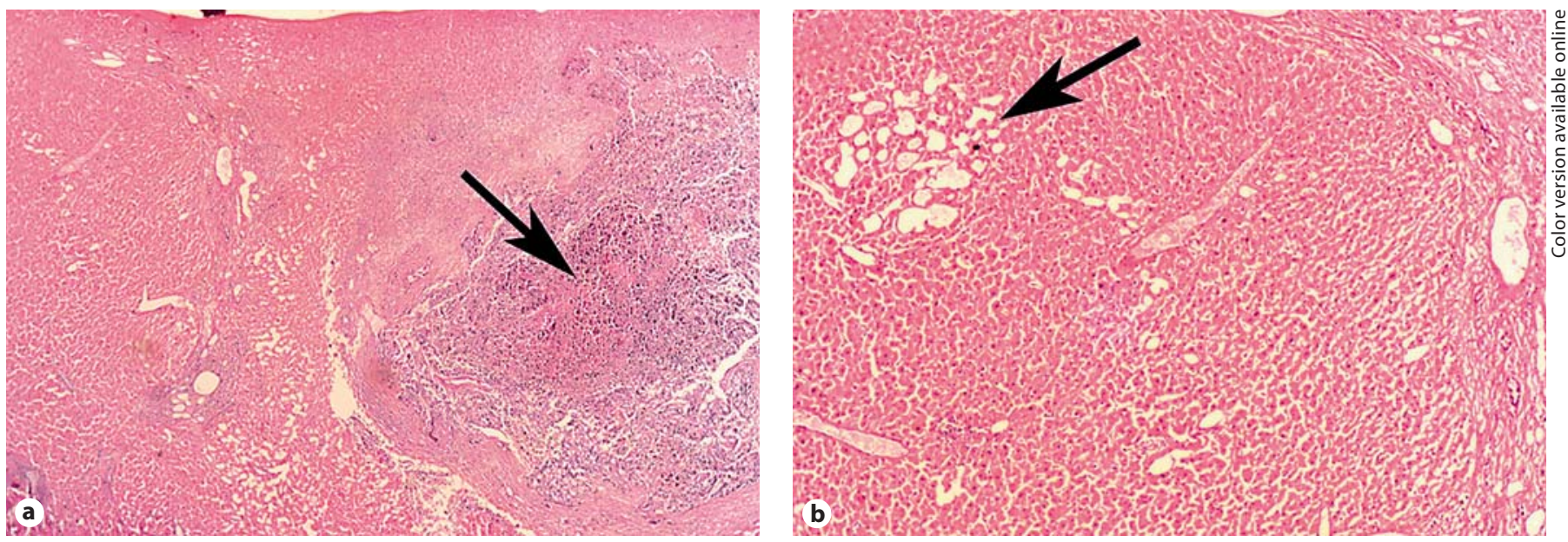

Fig. 3. Histology of the liver biopsy. Note the cancer-bearing area with central necrosis underlying the liver capsule (arrow; a) and scattered foci of dilatation of portal veins and sinusoids (arrow; b). Hematoxylin and eosin; $\times 10(\mathbf{a}), \times 40(\mathbf{b})$.

noma. Intramural or extramural vascular invasion was not detected. A metastatic tumor was found in the regional lymph nodes. The clinical stage of this rectal carcinoma was $\mathrm{T}_{3} \mathrm{~N}_{1} \mathrm{M}_{1}$. Chemotherapy with FOLFOX4 regimen (oxaliplatin, folinic acid, and 5-FU) was initiated after surgery. The patient has been well without evidence of recurrence for $>12$ months postoperatively.

\section{Discussion}

Hepar lobatum is a classic morphological entity that has been used synonymously with tertiary syphilitic liver because of its characteristic morbid anatomy at autopsy $[2,8]$. Hepar lobatum carcinomatosum (HLC) is the rarest form of metastatic liver disease. Busni [9] first introduced the term 'hepar lobatum carcinomatosum' in 1924. HLC is characterized by the combination of multifocal cancer-bearing scars and compensatory hyperplasia of the spared liver parenchyma. HLC may resemble its syphilitic counterpart, but tumor tissue can be demonstrated around or independently of those scar-like areas. Both scirrhous stromal reaction to carcinoma and vascular impairment due to infiltration have been suggested for the pathogenesis of multifocal cancer-bearing scars [10]. Metastatic liver cancers from the stomach, gallbladder, pancreas, etc. may commonly be associated with a scirrhous stroma, but HLC has not been routinely observed with such primaries.

The pathogenetic mechanism of HLC may be similar to that of hepar lobatum syphiliticum. In this respect, 2 further conditions may be required: inhomogeneous or diffuse but multifocal distribution of the affected blood vessels and slow progression of the process allowing the spared liver parenchyma to develop compensatory hypertrophy. Blockage of portal venous branches may cause Zahn's infarcts. Occlusion of hepatic venous branches may have a congestive effect, leading to its gradual shrinkage with fibrosis. Obstruction of the lymphatic vessels may add a dystrophic effect in terms of lymph stasis [10]. Gravel et al. [11] reported that, in their 2 cases of HLC associated with metastatic breast carcinoma, minute tumor deposits were seen mainly within sinusoidal spaces or within portal tracts along the junction with parenchyma. Occasional venules and/or lymphatics within fibrous septa contained intraluminal tumor cell clusters. However, we did not observe such changes in this case. In our patient, the only striking microscopic finding was focal areas of dilatation of portal veins and sinusoids which did not show any occlusion by tumor emboli. Moreover, triphasic helical CT of the liver revealed the portal and hepatic veins to be patent and no intrahepatic intravascular tumor thromboses or metastatic lesions were detected.

Hepar lobatum resulting from metastatic carcinoma is a very rare phenomenon, mostly documented in the setting of multiagent chemotherapy-treated breast carcinoma. Chin et al. [7] reported an unusual etiology for hepar lobatum which was related to the administration of chemotherapeutic agents (5-FU, adriamycin and mitomycin) resulting in the destruction of the tumor cells with consequent fissuring cicatrization in a patient who had gastric carcinoma with metastasis to the liver. In our case, 
however, the dose of 5-FU, the neoadjuvant chemotherapeutic agent used, was within the ordinary range, and repeated CT examinations prior to and after their administration demonstrated no alteration in liver morphology.

Many chemotherapeutic agents, including alkylating agents (cyclophosphamide), antimetabolites (methotrexate, 5-FU), cytotoxic antibiotics (doxorubicin, epirubicin, mitomycin, mitoxanthrone) and plant alkaloids (vinblastine, paclitaxel, docetaxel) have been used in patients reported to have developed HLC. Of these, no common denominator single agent was used in the treatment of patients who developed hepar lobatum. Except for methotrexate, none of the antineoplastic agents are known to induce significant hepatic fibrosis. Methotrexate can induce portal arachnoid fibrosis and sinusoidal collagen deposition occasionally leading to micronodular cirrhosis [12]. However, this patient with hepar lobatum who had received 5-FU in the neoadjuvant setting had no such pattern of fibrosis.

\section{Conclusion}

We described a case of liver dysmorphy revealed by metastatic rectal carcinoma. The liver morphology was misleading, mimicking tertiary syphilitic liver. When it is difficult to identify the cause of liver dysmorphy in a patient with known colorectal cancer, hepar lobatum carcinomatosum should be considered in the differential diagnosis.

\section{References}

1 Hahn RD: Syphilis of the liver. Am J Syph Gonorrhea Vener Dis 1943;27:529-561.

2 Symmers D, Spain DM: Hepar lobatum: clinical significance of the anatomic changes. Arch Pathol 1946;42:64-68.

3 Carson MJ, Chadwick DL, Brubaker CA, Cleland RS, Landing BH: Thirteen boys with progressive septic granulomatosis. Pediatrics 1965;35:405-412.

4 Ghon A: Leber: Die spezifischen Entzündungen; in Aschoff L (ed): Pathologische Anatomie: ein Lehrbuch für Studierende und Ärzte, ed 7. Jena, Fischer, 1928, pp 876882 .
Tsui WMS, Chow LTC: Advanced schistosomiasis as a cause of hepar lobatum. Histopathology 1993;23:495-497.

-6 Qizilbash A, Kontozoglou T, Sianos J, Scully K: Hepar lobatum associated with chemotherapy and metastatic breast cancer. Arch Pathol Lab Med 1987;111:58-61.

$\checkmark 7$ Chin NW, Chapman I, Jimenez FA: Complete chemotherapeutic regression of hepatic metastases with resultant hepar lobatum. Am J Gastroenterol 1987;82:149-151.

$\checkmark 8$ Shapiro E, Weiner H: The diagnosis of tertiary syphilis of the liver: 25 years after McCrae. Am J Med Sci 1951;222:494-499.
9 Busni NA: Hepar lobatum carcinomatosum. Virchows Arch A Pathol Anat Histopathol 1924;252:727-733.

10 Honma K: Hepar lobatum carcinomatosum due to metastatic breast carcinoma. Virchows Arch A Pathol Anat Histopathol 1987; 410:465-469.

11 Gravel DH, Begin LR, Brisson ML, Lamoureux E: Metastatic carcinoma resulting in hepar lobatum. Am J Clin Pathol 1996; 105:621627.

12 Nyfors A, Poulsen H: Morphogenesis of fibrosis and cirrhosis in methotrexate-treated patients with psoriasis. Am J Surg Pathol 1977;1:235-243. 